\title{
Abordaje de los probióticos en pediatría: el rol de Lactobacillus rhamnosus GG
}

a. Grupo de Trabajo en Gastroenterología y Nutrición Pediátrica, Hospital General de Agudos Dr. Ignacio Pirovano, Ciudad Autónoma de Buenos Aires, Argentina.

b. Departamento Materno Infantil, Hospital Universitario Austral, Pilar, Argentina.

c. Consultorios externos de Gastroenterología y Nutrición Pediátrica Hospital Pediátrico Federico Falcón, Pilar,

d. Servicio de Pediatría, Grupo Médico Las Lomas, San Isidro, Argentina.

e. Sanatorio de Niños de Rosario, Rosario, Argentina.

f. Instituto de Lactología Industrial, Facultad de Ingeniería Química, Universidad Nacional del Litoral, Santa Fe, Argentina.

Correspondencia: Gabriel Vinderola: gvinde@fiq.unl.edu.ar

Financiamiento:

Ninguno.

Conflicto de intereses: Mead Johnson Nutrition Argentina facilitó los encuentros y detalles técnicos del trabajo. El manuscrito fue escrito de forma independiente con la colaboración de todos los autores, sin tener la empresa control editorial alguno con respecto al resultado final. Los autores declaran que su única relación con Mead Johnson Nutrition Argentina fue haber participado de conferencias en simposios organizados por la empresa, de la misma forma que lo han hecho para otras empresas.

Recibido: 22-12-2020

Aceptado: 4-8-2021

\section{Approach to probiotics in pediatrics: the role of Lactobacillus rhamnosus GG}

\author{
Christian Boggio Marzet ${ }^{a}$ (D) Fernando Burgos $^{b}$ (D), Mónica Del Comparec (ID), \\ Ingrid Gerold $(\mathbb{D})$, Omar Tabacco ${ }^{(}$(D) Gabriel Vinderolaf $(\mathbb{D})$
}

\section{RESUMEN}

El parto prematuro, las cesáreas, los antibióticos y la lactancia materna limitada contribuyen al aumento de enfermedades crónicas no transmisibles. Elobjetivo fue realizar una revisión descriptiva del uso de probióticos en pediatría, con foco en la cepa Lactobacillus rhamnosus GG. Ciertos probióticos han demostrado ser eficaces en la diarrea aguda y en la diarrea asociada a antibióticos. L. rhamnosus GG y Saccharomyces boulardii pueden acortar la duración y los síntomas. L. reuteri DSM 17938 y L. rhamnosus GG fueron efectivos para el abordaje del cólico del lactante. El uso de esta cepa en fórmulas infantiles para alergia a las proteínas de leche de vaca promovería la adquisición más temprana de tolerancia. En la prevención de dermatitis atópica, la administración de L. rhamnosus GG durante el embarazo redujo su manifestación en el bebé. El empleo de probióticos como coadyuvantes es una posibilidad para considerar en la práctica pediátrica actual.

Palabras clave: microbiota, probióticos, fórmulas infantiles, Lactobacillus rhamnosus GG.

http: / / dx.doi.org/10.5546/ aap.2022.e1

Texto completo en inglés:

http: / / dx.doi.org/10.5546/ aap.2022.eng.e1

Cómo citar: Boggio Marzet, Burgos F, Del Compare M, Gerold I, et al. Abordaje de los probióticos en pediatría: el rol de Lactobacillus rhamnosus GG. Arch Argent Pediatr 2022;120(1):e1-e7.

\section{INTRODUCCIÓN}

La combinación de factores como el parto prematuro y el bajo peso al nacer, las cesáreas electivas, el uso de antibióticos en los dos primeros años de vida y la lactancia materna limitada contribuye al aumento de enfermedades crónicas no transmisibles en niños. ${ }^{1,2}$ Intervenciones microbianas tempranas, como aquellas que emplean probióticos, pueden ser una herramienta nutricional complementaria para el abordaje de algunas de estas patologías. ${ }^{3}$ Este trabajo se fundamenta en la posibilidad de ofrecer una actualización sobre el potencial benéfico del empleo de cultivos probióticos para la prevención y terapéutica de ciertas enfermedades crónicas que pueden aparecer en los primeros años de vida. El objetivo fue realizar una revisión descriptiva del uso de probióticos en pediatría, con foco en la cepa Lactobacillus rhamnosus GG (LGG).

El presente trabajo se encuadra dentro de la categoría Artículos especiales y es un trabajo de revisión y descripción narrativa de la temática objeto del artículo. Cada autor realizó una revisión a partir de materiales publicados indexados en Pubmed, seleccionando los trabajos que cada uno consideró de relevancia para ser incluidos en esta revisión. CBM desarrolló los aspectos relacionados a alergias; FB abordó los aspectos relacionados a los mecanismos de acción; MDC trató los aspectos de seguridad; IG expuso los efectos benéficos; OT explicó la colonización 
perinatal y el uso de probióticos en neonatología y GV planteó los aspectos microbiológicos. Las palabras claves utilizadas para la búsqueda bibliográfica fueron probiotics, microbiome, allergy, safety, perinatal colonization, infants, mechanisms of action, health benefits.

\section{COLONIZACIÓN INTESTINAL PERINATAL}

La visión de nuestra relación con los microorganismos ha cambiado y pasaron de considerarse exclusivamente patógenos a miembros fundamentales de un "entorno microbiano saludable" para el desarrollo del niño y su maduración inmunológica, metabólica y neurológica, ${ }^{4,5}$ desde el nacimiento.

La colonización perinatal es influenciada ya por el microbioma del aparato reproductor femenino, dominado por el género Lactobacillus. ${ }^{2}$ El ambiente uterino tampoco sería estéril. Se ha demostrado presencia microbiana en placenta, líquido amniótico y meconio, ${ }^{6}$ pero no es posible aún hablar de un "microbioma fetal".

El nacimiento y las primeras horas de vida definen el microbioma del lactante. ${ }^{7}$ Existen diferencias taxonómicas en el microbioma de niños nacidos por parto vaginal respecto a los nacidos por cesárea. La lactancia materna, idealmente instituida en la primera hora posterior al nacimiento, aporta microorganismos, oligosacáridos específicos y factores inmunológicos. ${ }^{8}$ Los efectos funcionales, no estrictamente nutricionales, de la lactancia materna son inmunológicos, madurativos y metabólicos, siendo el intermediario el microbioma del lactante.

El tipo de alimentación de la madre afecta la transmisión vertical microbiana. ${ }^{9}$ Los lactobacilos y las bifidobacterias constituyen los grupos microbianos más reconocidos como beneficiosos para el niño en este período por ser capaces de fermentar oligosacáridos de leche materna y generar un medio ácido en la luz intestinal que dificulta la colonización por patógenos; además, funcionan como mediadores del desarrollo del niño. ${ }^{1}$

Cuando los factores que promueven una adecuada colonización intestinal perinatal (parto a término, vaginal, lactancia materna) son sesgados por fenómenos como el parto prematuro o prácticas como la cesárea y la administración de antibióticos, la intervención microbiana mediante el uso de probióticos es una opción para el abordaje de la problemática generada por estos fenómenos.

\section{PROBIÓTICOS}

La Organización Mundial de la Salud estableció en 2002 que los probióticos son "microorganismos vivos que, cuando son administrados en cantidades suficientes, confieren un efecto benéfico sobre la salud del consumidor"..$^{10}$ Los probióticos pueden estar disponibles como suplementos dietarios o en alimentos fermentados (yogures) o no (fórmulas infantiles), ${ }^{11}$ donde es posible verificar su viabilidad. ${ }^{12}$

Los probióticos deben tener identidad microbiológica (género, especie y cepa); ser seguros en los sujetos donde se prevé administrar la cepa; estar viables al ser administrados; y disponer de estudios de eficacia que demuestren sus efectos benéficos. ${ }^{13}$ Los efectos son cepadependientes. Por ejemplo, L. rhamnosus CRL1505 y L. rhamnosus GG son cepas diferentes. La primera es utilizada en un programa social escolar y posee eficacia para la prevención de infecciones del árbol respiratorio. ${ }^{14} \mathrm{~L}$. rhamnosus GG, aislada por Goldin y Gorbach, ${ }^{15}$ es la cepa con mayor cantidad de estudios clínicos de eficacia;, ${ }^{16}$ el primero se registró en 1995 para el tratamiento de gastroenteritis aguda por rotavirus. ${ }^{17}$

En numerosos casos, los efectos benéficos de los probióticos se observan sin que ocurran modificaciones significativas en la composición o en la función de la microbiota intestinal, debido a que los mecanismos de acción por los cuales pueden ejercer sus beneficios son múltiples. ${ }^{18}$

\section{SEGURIDAD DE LOS PROBIÓTICOS}

La evaluación de la seguridad de una cepa es una etapa previa, y obligatoria, a la determinación de sus propiedades probióticas, que considera su identidad microbiológica, la ruta de administración, la dosis y el estado de salud del grupo poblacional que la recibe. ${ }^{19}$

En términos generales, los lactobacilos son microorganismos seguros, se encuentran en el listado positivo de microorganismos con estatus QPS (presunción cualificada de seguridad, por su sigla en inglés) de la Agencia Europea de Seguridad Alimentaria. ${ }^{20}$ El riesgo de infección por estos microorganismos se estimó en aproximadamente un caso por cada 10 millones de personas..$^{21}$ Numerosos estudios han demostrado la seguridad de L. rhamnosus GG, incluso en pacientes inmunodeprimidos (prematuros, con enfermedades inflamatorias intestinales, con infección por el virus de la inmunodeficiencia humana o adultos mayores, entre otros). ${ }^{22}$ 
Hay pacientes inmunocomprometidos graves (pancreatitis aguda, aquellos sometidos a cirugías gastrointestinales mayores) que podrían beneficiarse del uso de probióticos, ya que son susceptibles a sepsis, síndrome de respuesta inflamatoria sistémica o falla multiorgánica. Sin embargo, el reporte en la literatura de ciertos efectos adversos en estos pacientes ${ }^{23}$ hace necesario que la seguridad sea demostrada para cada cepa en particular, en la patología considerada. No se aconseja el uso de probióticos en pacientes con acceso venoso central, síndrome de intestino corto o enfermedades de las válvulas cardíacas. ${ }^{23}$

Debido a la posibilidad de transferencia de resistencia a los antibióticos desde cepas administradas oralmente a microorganismos residentes de la microbiota intestinal, se utilizan tecnologías moleculares para asegurar la ausencia de genes de transferencia de resistencia a antibióticos en cepas candidatas a ser probióticos. ${ }^{24}$

L. rhamnosus $\mathrm{GG}^{\circledR}$ es considerada segura por la Administración de Medicamentos y Alimentos de los Estados Unidos (FDA, por su sigla en inglés). En Europa, las cepas pertenecientes a la especie L. rhamnosus poseen el estatus QPS y se permite su uso como ingrediente en fórmulas infantiles. Todas las cepas de L. rhamnosus, incluida la LGG, son sensibles a la mayoría de los antibióticos de uso clínico, aunque son resistentes a la vancomicina, aunque esto es un factor inherente, no transmisible. ${ }^{25}$

En poblaciones alérgicas a las proteínas de la leche o el huevo, el riesgo de que se produzca una reacción alérgica no depende del probiótico en sí, sino de los excipientes utilizados en la formulación del producto. En ciertos productos comercializados con probióticos, se encontraron trazas de alérgenos no advertidas en el etiquetado. ${ }^{26}$ El Comité de Nutrición, Hepatología y Nutrición de la Sociedad Europea de Gastroenterología Pediátrica (ESPGHAN, por su sigla en inglés) y el Grupo de Trabajo ESPGHAN para Probióticos y Prebióticos se han pronunciado sobre el uso de cepas específicas y sobre los aspectos de seguridad que deben considerarse antes de la administración. ${ }^{27}$

\section{IMPACTO DE LOS PROBIÓTICOS EN SALUD}

En los últimos años se ha incrementado el interés científico en los beneficios de diferentes cepas de probióticos para la prevención y tratamiento de trastornos gastrointestinales, enfermedades metabólicas, ${ }^{28}$ prevención y tratamiento de alergias, ${ }^{29}$ y la modulación del sistema inmune en general. ${ }^{30}$ En pediatría, se ha demostrado la efectividad de ciertos probióticos en el tratamiento de gastroenteritis agudas, ${ }^{31}$ en la prevención y tratamiento de la diarrea asociada a antibióticos, ${ }^{32}$ de cólicos del lactante ${ }^{33}$ y de eccema atópico. $^{34}$

Ciertas cepas de Saccharomyces boulardii, L. casei y L. rhamnosus han demostrado ser eficaces en el tratamiento de diarrea aguda en niños, sobre todo en las producidas por rotavirus, acortando en un día la duración. ${ }^{35,36}$ En relación con la diarrea asociada a antibióticos, los probióticos con mayor evidencia científica son LGG y $S$. boulardii. Su administración reduciría en un $51 \%$ el riesgo de diarrea si se comienzan a administrar desde el mismo día del inicio del tratamiento con antibióticos.

En relación con el cólico del lactante, en un metaanálisis realizado con la administración diaria de L. reuteri DSM 17938, se observó una disminución significativa del tiempo de llanto de 51 minutos por día. ${ }^{37}$ LGG también demostró eficacia luego de su administración durante 28 días, disminuyendo a la mitad los días de llanto. $^{38}$

La utilidad de LGG para estos trastornos gastrointestinales se debe a que es estable frente a la acidez gástrica y a la bilis, y puede realizar su actividad benéfica a nivel intestinal.

Respecto a la obesidad y al síndrome metabólico, se demostró la efectividad de la administración de probióticos de las especies L. plantarum y L. rhamnosus en el mejoramiento del perfil lipídico, reducción del tejido adiposo, disminución del colesterol total sérico, LDL colesterol y triglicéridos, y aumento del HDL

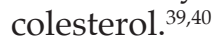

En las últimas décadas, se ha observado un aumento de casos de alergias alimentarias, respiratorias y dermatológicas, que coincide con una drástica disminución de la diversidad y abundancia de la microbiota intestinal, relacionada a la vez con el aumento de partos prematuros, cesáreas, la reducción de la lactancia materna, el uso de antibióticos, la menor exposición al medioambiente y la excesiva higiene. Apoyando esta teoría de la higiene, los datos epidemiológicos muestran la menor incidencia de enfermedades alérgicas en poblaciones rurales con respecto a poblaciones urbanas. ${ }^{41}$ En este contexto, se dirige la atención al uso de probióticos para la prevención y el 
tratamiento de asma, eccema atópico y alergias alimentarias. Los factores que contribuyen a tener enfermedades atópicas son la alteración de la barrera de la piel y de la mucosa intestinal.

El sistema inmunológico del recién nacido está fuertemente dominado por células T helper tipo 2 $\left(T_{h} 2\right)$, y va cambiando gradualmente la respuesta inmunitaria hacia células $\mathrm{T}$ helper tipo $1\left(\mathrm{~T}_{h} 1\right)$ para mantener la homeostasis. El fenotipo $T_{h} 2$ conduce a niveles mayores de inmunoglobulina E (IgE) y a la activación de mastocitos, lo que a su vez aumenta la susceptibilidad a los trastornos alérgicos. La microbiota juega un papel determinante en el balance de las respuestas inmunes $\mathrm{T}_{\mathrm{h}} 1 / \mathrm{T}_{\mathrm{h}} 2 .{ }^{42}$

La patogenia general de una respuesta alérgica implica la sensibilización a alérgenos (polen, alimentos, etc.), lo cual induce una respuesta inflamatoria transitoria, con aumento de IgE específica de alérgenos y migración de células $\mathrm{T}$ efectoras, mastocitos y eosinófilos al sitio de exposición. Los eosinófilos desempeñan un papel importante en las alergias respiratorias y digestivas por desgranulación en el sitio de exposición, lo que provoca el reclutamiento de células inmunitarias y daño tisular. La mayoría de los antígenos encontrados en el tracto gastrointestinal provienen de la dieta y de la microbiota intestinal, los cuales pueden afectar la tolerancia inmune, siendo la presentación antigénica de estos factores dietarios a las células $\mathrm{T}$ reguladoras, un paso crucial para evitar una respuesta inmune a los antígenos alimentarios. ${ }^{43}$ Las alteraciones en los niveles o en la diversidad de la microbiota pueden alterar la tolerancia inmunológica de la mucosa, lo cual conduce a enfermedades alérgicas alimentarias y respiratorias. ${ }^{44}$ Por otro lado, niveles bajos de $\operatorname{IgA}$ en la superficie de la barrera intestinal también pueden contribuir a las alergias alimentarias. Niveles bajos de IgA y la microbiota están relacionados: la microbiota intestinal puede estimular las células dendríticas en las placas de Peyer para activar las células B, lo cual conduce a la producción de anticuerpos IgA específicos. Esta estimulación puede ocurrir a través de la producción de ácidos grasos de cadena corta por parte de la microbiota. ${ }^{45}$

La microbiota intestinal también puede influir en el desarrollo de la tolerancia oral y la sensibilización a los antígenos alimentarios. Una mayor abundancia de clostridios y de especies del filo Firmicutes se relacionó con la alergia a la proteína de leche de vaca (APLV) en bebés. La administración de fórmulas de caseína suplementadas con LGG en bebés con APLV condujo al enriquecimiento de especies productoras de butirato, promoviendo la tolerancia. La suplementación con LGG reduce el riesgo de otras manifestaciones alérgicas. ${ }^{46}$

L. rhamnosus GG es uno de los probióticos más estudiados para estas patologías. Para evaluar si la dermatitis atópica podría ser prevenida en los primeros años de vida, se realizó un estudio con 159 madres embarazadas con historia familiar de alergias. Las participantes recibieron LGG $\left(10^{10} \mathrm{UFC}\right)$ o placebo, diariamente durante 2-4 semanas antes del parto. Luego del nacimiento se administró la cepa a la madre, con lactancia exclusiva, o bien al bebé durante 6 meses. Se observó una disminución significativa de la incidencia de eccema atópico en el grupo que recibió LGG. ${ }^{34}$ Los hijos de madres que recibieron LGG presentaron menor riesgo de desarrollar dermatitis atópica durante los dos primeros años de vida. ${ }^{47}$

Aun con este grado de evidencia, se necesitarían mayor cantidad de estudios, ya que una revisión sistémica de 29 estudios clínicos determinó que no hay evidencia suficiente aún para recomendar el empleo de probióticos en la prevención primaria de enfermedades alérgicas, como asma o rinitis alérgica. Sin embargo, sugirieron el uso en mujeres embarazadas/ lactantes y en bebés con antecedentes familiares de enfermedad alérgica. ${ }^{48}$

\section{PROBIÓTICOS EN PREMATUROS}

En los últimos años, la industria productora de fórmulas infantiles ha direccionado su esfuerzo en la investigación y desarrollo de los principales factores responsables de los efectos funcionales de la leche materna (oligosacáridos, microorganismos y sus metabolitos), y el agregado de probióticos y prebióticos ha demostrado ser beneficioso en la prevención de trastornos alérgicos en la infancia. ${ }^{16}$

El recién nacido prematuro es un modelo adecuado de vulneración de los factores positivos para la generación de un microbioma intestinal saludable, debido a la inmadurez intestinal, la cesárea, el uso de antibióticos y la alimentación heteróloga, por lo cual se han estudiado estrategias nutricionales para mejorarlo, ${ }^{49,50}$ entre ellas el uso de probióticos. Una revisión sistemática con metaanálisis del uso de probióticos en prematuros con muy bajo peso (30 estudios aleatorizados y 14 estudios 
observacionales) demostró resultados favorables en todas las variables analizadas (menor desarrollo de enterocolitis graves, sepsis tardías y mortalidad), aunque estos trabajos se realizaron utilizando distintos microorganismos, solos o combinados, y con dosis variables. ${ }^{51}$

Otra revisión sistemática, en 4527 recién nacidos menores de 37 semanas de gestación, mostró resultados favorables del uso de probióticos en la reducción del tiempo de alimentación enteral exclusiva. El trabajo demostró también efectos positivos en la ganancia de peso y en el flujo mesentérico posprandial, pero también se utilizaron una diversidad de cepas y dosis, lo que no permite generalizar las conclusiones a todos los probióticos. ${ }^{52} \mathrm{No}$ obstante, existe consenso sobre la ausencia de complicaciones derivadas del uso de probióticos en prematuros, por lo cual, el amplio margen de seguridad es un aspecto muy importante para considerar.

La utilización de LGG en 640 recién nacidos de muy bajo peso (menor a $1500 \mathrm{~g}$ ), de un mismo servicio y durante 8 años, mostró una mayor incidencia de enterocolitis necrosante (ECN) grave luego del uso de esta cepa $(10,2 \%$ vs. $16,8 \%$ ), sin encontrarse episodios de sepsis. ${ }^{53}$

En una reciente publicación, la ESPGHAN, a través de su grupo de expertos en prebióticos y probióticos y de su Comité de Nutrición, fijó una posición cautelosa en la utilización de probióticos en prematuros, considerando la evidencia aún poco robusta. Además, plantea la necesidad de discriminar qué tipo de probiótico debe ser utilizado, a partir de qué momento, la concentración, el vehículo (leche de madre o fórmula), y pone énfasis en la necesidad de nuevos estudios. ${ }^{54}$ En contraposición, en un reporte también reciente de la Asociación Americana de Gastroenterología, se recomienda el uso de probióticos en recién nacidos prematuros menores de 37 semanas y de bajo peso, incluido LGG entre las cepas recomendadas, analizando parámetros como la incidencia de ECN, mortalidad general, sepsis tardía y estancia en el servicio de neonatología. ${ }^{55}$

En definitiva, la evidencia actual es muy promisoria en relación con el uso de probióticos en neonatología, en recién nacidos prematuros, con el objetivo de disminuir la incidencia de las principales complicaciones, tales como la ECN, la sepsis tardía y la mortalidad general, con márgenes confiables de seguridad. No obstante, falta aún determinar específicamente cuál probiótico, solo o en combinación, debe ser usado, el momento de inicio y finalización del tratamiento, y la dosis adecuada. Además, sigue siendo todavía un enigma la utilización en los neonatos de muy bajo peso, menor a $1000 \mathrm{~g}$.

El microbioma del recién nacido prematuro es una comunidad enorme de microorganismos que se desarrollan de manera simbiótica entre sí y en estrecha relación con el sistema inmunológico intestinal. Más allá del potencial uso de probióticos, las prácticas neonatológicas generales en el tratamiento de estos pacientes deben ser revisadas globalmente para facilitar el desarrollo de las especies bacterianas más saludables.

\section{MECANISMOS DE ACCIÓN DE LOS PROBIÓTICOS}

Los probióticos pueden influenciar el ecosistema intestinal al actuar sobre los mecanismos inmunológicos de la mucosa, interactuar con microorganismos comensales o potencialmente patógenos, generar productos metabólicos, o comunicarse con las células del huésped utilizando señales químicas, producto de su metabolismo. Se estima que estos fenómenos median la mayoría de los efectos benéficos descriptos para los probióticos.

Entre los mecanismos de acción más relevantes de los probióticos, se pueden destacar la resistencia a la colonización por patógenos, ya que pueden ocupar temporalmente nichos funcionales que quedan vacantes en la microbiota residente, previniendo infecciones oportunistas, o pueden modificar el ambiente local a través de la producción de ácidos grasos de cadena corta (AGCC), ácido láctico, bacteriocinas y especies reactivas de oxígeno para inhibir el crecimiento de patógenos. ${ }^{56}$

Ciertos probióticos contribuyen a la mayor disponibilidad de vitamina $\mathrm{K}, \mathrm{B}_{12}$, piridoxina, biotina, ácido fólico, ácido nicotínico y tiamina. ${ }^{57}$ Algunas cepas pueden considerarse inmunoestimuladoras por su capacidad para inducir inmunidad mediada por células NK (asesinas naturales, por su sigla en inglés). ${ }^{58}$ Otras pueden considerarse antinflamatorias, debido a su capacidad para inducir interleucinas IL-10. ${ }^{59}$ Los probióticos además pueden activar macrófagos locales, aumentando la presentación de antígenos a linfocitos B, estimulando la secreción de IgA. ${ }^{60}$

En particular, entre los mecanismos de acción descriptos para L. rhamnosus GG, se puede mencionar que los polisacáridos y pelos (pilli) presentes en su superficie le permiten adherirse 
y colonizar temporalmente la mucosa intestinal. LGG es capaz de producir tanto una biopelícula que puede proteger mecánicamente la mucosa como diferentes factores solubles beneficiosos para el intestino al aumentar la supervivencia de la cripta intestinal, disminuir la apoptosis del epitelio intestinal y preservar la integridad del citoesqueleto. Además, LGG, gracias a su proteína lectina 1 y 2, inhibe algunos patógenos como Salmonella. Esta cepa es capaz de estimular la respuesta inmunológica inespecífica mediada por IgA, IgG e IgM, y la respuesta inmunológica celular dependiente de linfocitos T CD4+, es capaz a la vez de promover la respuesta inmunológica $T_{h} 1$, reduciendo la expresión de varios marcadores de activación e inflamación en los monocitos y aumentando la producción de interleucinas IL-10, IL-12 y el factor de necrosis tumoral- $\alpha$ en macrófagos. ${ }^{61}$

\section{CONCLUSIONES}

El parto prematuro, el bajo peso al nacer, las cesáreas electivas, el uso de antibióticos y la lactancia materna limitada, entre otros factores, contribuyeron al aumento de enfermedades crónicas no transmisibles en niños. El uso de probióticos surge como una estrategia microbiológica segura en pediatría para la promoción de la inmunidad intestinal.

L. rhamnosus GG (LGG) y S. boulardii han demostrado ser seguros y eficaces en la prevención de la diarrea aguda y la diarrea asociada a antibióticos, acortando la duración y disminuyendo los síntomas. En el caso del cólico del lactante, tanto L. reuteri DSM 17938 como LGG son capaces de disminuir significativamente el tiempo de duración. En la prevención de dermatitis atópica, la administración de LGG durante el embarazo demostró una reducción en su manifestación en el bebé.

Si bien se necesita mayor evidencia sobre los efectos terapéuticos y preventivos de los probióticos sobre las alergias alimentarias, los estudios actualmente disponibles sugerirían que el uso de LGG en fórmulas infantiles para APLV promovería la adquisición más temprana de tolerancia.

\section{REFERENCIAS}

1. Milani C, Duranti S, Bottacini F, Casey E, et al. The First Microbial Colonizers of the Human Gut: Composition, Activities, and Health Implications of the Infant Gut Microbiota. Microbiol Mol Biol Rev. 2017; 81:e00036-17.

2. Selma-Royo M, Tarrazó M, García-Mantrana I, GómezGallego C,et al. Shaping Microbiota During the First 1000 Days of Life. Adv Exp Med Biol. 2019; 1125:3-24.
3. Salminen S, Stahl B, Vinderola G, Szajewska H. Infant formula supplemented with biotics: Current knowledge and future perspectives. Nutrients. 2020; 12(7):1952.

4. Douglas-Escobar M, Elliott E, Neu J. Effect of intestinal microbial ecology on the developing brain. JAMA Pediatr. 2013; 167(4):374-9.

5. Sommer F, Bäckhed F. The gut microbiota-masters of host development and physiology. Nat Rev Microbiol. 2013; 11(4):227-38.

6. Stinson LF, Boyce MC, Payne MS, Keelan JA. The not-sosterile womb: Evidence that the human fetus is exposed to bacteria prior to birth. Front Microbiol. 2019; 10:1124.

7. Rutayisire E, Huang K, Liu Y, Tao F. The mode of delivery affects the diversity and colonization pattern of the gut microbiota during the first year of infants' life: a systematic review. BMC Gastroenterol. 2016; 16(1):86.

8. Pannaraj PS, Li F, Cerini C, Bender JM, et al. Association between breast milk bacterial communities and establishment and development of the infant gut microbiome. JAMA Pediatr. 2017; 171(7):647-54.

9. Rodríguez JM, Murphy K, Stanton C, Ross RP, et al. The composition of the gut microbiota throughout life, with an emphasis on early life. Microb Ecol Health Dis. 2015; 26:26050.

10. HillC, Guarner F, Reid G, Gibson GR, etal.Expert consensus document: The international scientific association for probiotics and prebiotics consensus statement on the scope and appropriate use of the term probiotic. Nat Rev Gastroenterol Hepatol. 2014; 11(8):506-14.

11. Sanders ME, Marco ML. Food formats for effective delivery of probiotics. Annu Rev Food Sci Technol. 2010; 1:65-85.

12. Vinderola G, Reinheimer J, Salminen S. The enumeration of probiotic issues: From unavailable standardised culture media to a recommended procedure? Int Dairy J. 2019; 96:58-65.

13. Binda S, Hill C, Johansen E, Obis D, et al. Criteria to Qualify Microorganisms as "Probiotic" in Foods and Dietary Supplements. Front Microbiol. 2020; 11:1662.

14. Villena JC, Salva MS, Nuñez MS, Corzo J, et al. Probiotics for Everyone! The Novel Immunobiotic Lactobacillus rhamnosus CRL1505 and the Beginning of Social Probiotic Programs in Argentina. Int J Biotechnol Wellness Ind. 2012; 1(3):189-98.

15. Pace F, Pace M, Quartarone G. Probiotics in digestive diseases: Focus on Lactobacillus GG. Minerva Gastroenterol Dietol. 2015; 61(4):273-92.

16. Dronkers TMG, Ouwehand AC, Rijkers GT. Global analysis of clinical trials with probiotics. Heliyon. 2020; 6(7):e04467.

17. Majamaa H, Isolauri E, Saxelin M, Vesikari T. Lactic acid bacteria in the treatment of acute rotavirus gastroenteritis. J Pediatr Gastroenterol Nutr. 1995; 20(3):333-8.

18. Wieërs G, Belkhir L, Enaud R, Leclercq S, et al. How Probiotics Affect the Microbiota. Front Cell Infect Microbiol. 2020; 9:454.

19. Sanders ME, Akkermans LMA, Haller D, Hammerman C, et al. Safety assessment of probiotics for human use. Gut Microbes. 2010; 1(3):164-85.

20. EFSA Panel on Biological Hazards (BIOHAZ), Ricci A, Allende A, Bolton D, et al. Scientific Opinion on the update of the list of QPS $\square$ recommended biological agents intentionally added to food or feed as notified to EFSAt. EFSA J. 2017; 15(3): $\mathrm{e} 04664$.

21. Bernardeau M, Guguen M, Vernoux JP. Beneficial lactobacilli in food and feed: Long-term use, biodiversity and proposals for specific and realistic safety assessments. FEMS Microbiol Rev. 2006; 30(4):487-513.

22. Bernardeau M, Vernoux JP, Henri-Dubernet $S$, Guéguen M. Safety assessment of dairy microorganisms: the Lactobacillus genus. Int J Food Microbiol. 2008; 126(3):27885.

23. Hojsak I, Fabiano V, Pop TL, Goulet O, et al. Guidance on the use of probiotics in clinical practice in children with 
selected clinical conditions and in specific vulnerable groups. Acta Paediatr. 2018; 107(6):927-37.

24. Hempel S, Newberry S, Ruelaz A, Wang Z, et al. Safety of probiotics used to reduce risk and prevent or treat disease. Evid Rep Technol Assess (Full Rep). 2011; (200):1-645.

25. Tynkkynen S, Singh K V, Varmanen P. Vancomycin resistance factor of Lactobacillus rhamnosus GG in relation to enterococcal vancomycin resistance (van) genes. Int J Food Microbiol. 1998; 41(3):195-204.

26. Martín-Muñoz MF, Fortuni M, Caminoa M, Belver T, et al. Anaphylactic reaction to probiotics: Cow's milk and hen's egg allergens in probiotic compounds. Pediatr Allergy Immunol. 2012; 23(8):778-84.

27. Braegger C, Chmielewska A, Decsi T, Kolacek S, et al. Supplementation of infant formula with probiotics and/ or prebiotics: A systematic review and comment by the ESPGHAN committee on nutrition. J Pediatr Gastroenterol Nutr. 2011; 52(2):238-50.

28. Choi S, Hwang YJ, Shin MJ, Yi H. Difference in the gut microbiome between ovariectomy-induced obesity and diet-induced obesity. J Microbiol Biotechnol. 2017; 27(12):2228-36.

29. Mennini M, Dahdah L, Artesani MC, Fiocchi A, Martelli A. Probiotics in asthma and allergy prevention. Front Pediatr. 2017; 5:165.

30. Guarner F, Requena T, Marcos A. Consensus statements from the workshop "Probiotics and health: Scientific evidence". Nutr Hosp. 2010; 25(5):700-4.

31. Schnadower D, Tarr PI, Casper TC, Gorelick MH, et al. Lactobacillus rhamnosus GG versus Placebo for Acute Gastroenteritis in Children. NEngl JMed.2018;379(21):200214.

32. Szajewska H, Canani RB, Guarino A, Hojsak I, et al. Probiotics for the prevention of antibiotic-associated diarrhea in children. J Pediatr Gastroenterol Nutr. 2016; 62(3):495-506.

33. Anabrees J, Indrio F, Paes B, AlFaleh K. Probiotics for infantile colic: A systematic review. BMC Pediatr. 2013; 13:186.

34. Kalliomäki M, Salminen S, Arvilommi H, Kero P, et al. Probiotics in primary prevention of atopic disease: A randomised placebo-controlled trial. Lancet. 2001; 357(9262):1076-9.

35. Dinleyici EC, Eren M, Ozen M, Yargic ZA, Vandenplas Y. Effectiveness and safety of Saccharomyces boulardii for acute infectious diarrhea. Expert Opin Biol Ther. 2012; 12(4):395-410.

36. Szajewska H, Wanke M, Patro B. Meta-analysis: The effects of Lactobacillus rhamnosus GG supplementation for the prevention of healthcare-associated diarrhoea in children. Aliment Pharmacol Ther. 2011; 34(9):1079-87.

37. Sung V, D'AmicoF, Cabana MD, Chau K, et al. Lactobacillus reuteri to treat infant colic: a meta-analysis. Pediatrics. 2018; 141(1):e20171811.

38. Savino F, Montanari P, Galliano I, Daprà V, Bergallo M. Lactobacillus rhamnosus GG (ATCC 53103) for the management of infantile colic: a randomized controlled trial. Nutrients. 2020; 12(6):1693.

39. Torres S, Fabersani E, Marquez A, Gauffin-Cano P. Adipose tissueinflammation and metabolic syndrome. The proactive role of probiotics. Eur J Nutr. 2019; 58(1):27-43.

40. Ettinger G, MacDonald K, Reid G, Burton JP. The influence of the human microbiome and probiotics on cardiovascular health. Gut Microbes. 2014; 5(6):719-28.

41. Daley D. The evolution of the hygiene hypothesis: The role of early-life exposures to viruses and microbes and their relationship to asthma and allergic diseases. Curr Opin Allergy Clin Immunol. 2014; 14(5):390-6.

42. Walker WA, Iyengar RS. Breast milk, microbiota, and intestinal immune homeostasis. Pediatr Res. 2015; 77(12):220-8.
43. Kim KS, Hong SW, Han D, Yi J, et al. Dietary antigens limit mucosal immunity by inducing regulatory $\mathrm{T}$ cells in the small intestine. Science. 2016; 351(6275):858-63.

44. Abrahamsson TR, Jakobsson HE, Andersson AF, Björkstén $\mathrm{B}$, et al. Low diversity of the gut microbiota in infants with atopic eczema. J Allergy Clin Immunol. 2012; 129(2):434-40. e1-2.

45. Suzuki K, Kawamoto S, Maruya M, Fagarasan S. GALT: Organization and dynamics leading to IgA synthesis. Adv Immunol. 2010; 107:152-85.

46. Berni Canani R, Di Costanzo M, Bedogni G, Amoroso A, et al. Extensively hydrolyzed casein formula containing Lactobacillus rhamnosus GG reduces the occurrence of other allergic manifestations in children with cow's milk allergy: 3-year randomized controlled trial. J Allergy Clin Immunol. 2017; 139(6):1906-13.e4.

47. Rautava S, Kalliomäki M, Isolauri E. Probiotics during pregnancy and breast-feeding might confer immunomodulatory protection against atopic disease in the infant. J Allergy Clin Immunol. 2002; 109(1):119-21.

48. Cuello-García CA, Brozek JL, Fiocchi A, Pawankar R, et al. Probiotics for the prevention of allergy: A systematic review and meta-analysis of randomized controlled trials. J Allergy Clin Immunol. 2015; 136(4):952-61.

49. Mesa MD, Loureiro B, Iglesia I, Fernández González S, et al. The evolving microbiome from pregnancy to early infancy: a comprehensive review. Nutrients. 2020; 12(1):133.

50. Diggikar S. Neonatal microbiome: A complex, invisible organ and its evolving role in neonatal illness and beyond. J Clin Neonatol. 2019; 8(1):5-9.

51. Dermyshi E, Wang Y, Yan C, Hong W, et al. The "Golden Age" of Probiotics: a systematic review and meta-analysis of randomized and observational studies in preterm infants. Neonatology. 2017; 112(1):9-23.

52. Athalye-Jape G, Deshpande G, Rao S, Patole S. Benefits of probiotics on enteral nutrition in preterm neonates: A systematic review. Am J Clin Nutr. 2014; 100(6):1508-19.

53. Kane AF, Bhatia AD, Denning PW, Shane AL, Patel RM. Routine Supplementation of Lactobacillus rhamnosus GG and Risk of Necrotizing Enterocolitis in Very Low Birth Weight Infants. J Pediatr. 2018; 195:73-9.e2.

54. van den Akker CHP, van Goudoever JB, Shamir R, Domellöf $\mathrm{M}$, et al. Probiotics and Preterm Infants: A Position Paper by the European Society for Paediatric Gastroenterology Hepatology and Nutrition Committee on Nutrition and the European Society for Paediatric Gastroenterology Hepatology and Nutrition Working Group for Pr. J Pediatr Gastroenterol Nutr. 2020; 70(5):664-80.

55. Su GL, Ko CW, Bercik P, Falck-Ytter Y, et al. AGA Clinical Practice Guidelines on the role of probiotics in the management of gastrointestinal disorders. Gastroenterology. 2020; 159(2):697-705.

56. Plaza-Diaz J, Ruiz-Ojeda FJ, Gil-Campos M, Gil A. Mechanisms of Action of Probiotics. Adv Nutr. 2019; 10(Suppl 1):S49-66.

57. Gu Q, Li P. Biosynthesis of Vitamins by Probiotic Bacteria. In: Rao V, Rao L (eds). Probiotics and Prebiotics in Human Nutrition and Health. Rijeka: IntechOpen; 2016.Págs.13548.

58. Aziz N, Bonavida B. Activation of natural killer cells by probiotics. For Immunopathol Dis Therap. 2016; 7(1-2):41-55.

59. Azad MAK, Sarker M, Li T, Yin J. Probiotic Species in the Modulation of Gut Microbiota: An Overview. Biomed Res Int. 2018; 2018:9478630.

60. Ohland CL, MacNaughton WK. Probiotic bacteria and intestinal epithelial barrier function. Am J Physiol Gastrointest Liver Physiol. 2010; 298(6):G807-19.

61. Capurso L. Thirty Years of Lactobacillus rhamnosus GG A Review. J Clin Gastroenterol. 2019; 53 (Suppl 1):S1-41. 